\title{
O AJUSTAMENTO PSICOSSOCIAL E A QUALIDADE DE VIDA DE PA- CIENTES SUBMETIDOS AO TRANSPLANTE DE MEDULA ÓSSEA (TMO)
}

\author{
PSYCHOSOCIAL ADJUSTMENT AND QUALITY OF LIFE OF PATIENTS \\ SUBMITTED TO BONE MARROW TRANSPLANTATION (BMT)
}

Ângela da Costa Almeida1', Sonia Regina Loureiro², Júlio César Voltarelli

\begin{abstract}
${ }^{1}$ Mestranda em Psicologia da Faculdade de Filosofia, Ciências e Letras de Ribeirão Preto da Universidade de São Paulo; ${ }^{2}$ Docente do Departamento de Neurologia, Psiquiatria e Psicologia Médica; ${ }^{3}$ Docente do Departamento de Clínica Médica e Supervisor da Unidade de Transplante de Medula Óssea do Hospital das Clínicas - Faculdade de Medicina de Ribeirão Preto da Universidade de São Paulo. CorRESPONDÊNCIA: Profa. Dra. Sonia Regina Loureiro - Departamento de Neurologia, Psiquiatria e Psicologia Médica da FMRP- USP.; Av. Nove de Julho, 980 - Jd. Sumaré - CEP: 14025-000 - Ribeirão Preto - SP.
\end{abstract}

ALMEIDA AC; LOUREIRO SR \& VOLTARELLI JC. O ajustamento psicossocial e a qualidade de vida de pacientes submetidos ao transplante de medula óssea (TMO). Medicina, Ribeirão Preto, 31: 296-304, abr./jun. 1998.

RESUMO: O presente estudo tem como objetivo avaliar as condições psicológicas e a qualidade de vida de pacientes submetidos ao transplante de medula óssea (TMO), em dois momentos de recuperação - adaptação inicial e adaptação a longo prazo - visando uma compreensão dos recursos disponíveis para a adaptação e o ajustamento psicossocial.

UNITERMOS: Transplante de Medula Óssea. Controle Interno-Externo. Ajustamento Social. Avaliação Psicológica.

\section{INTRODUÇÃO}

O Transplante de Medula Óssea (TMO), enquanto modalidade terapêutica, tem implicações físicas e psicossociais que só mais recentemente têm sido estudadas. Entre os componentes que avaliam a qualidade de vida dos pacientes submetidos ao TMO, em geral, estão o desempenho físico, o estado psicológico e a interação social ${ }^{(1,2,3)}$. Apesar de as complicações médicas serem bem documentadas na literatura, pouco se sabe sobre o funcionamento psicossocial associado à sobrevida de pacientes submetidos a TMO, especialmente no que diz respeito aos recursos pessoais e de estrutura da personalidade.

Pesquisas recentes ${ }^{(4 / 7)}$ têm registrado uma diversidade de efeitos psicossociais do TMO, incluindo disfunção sexual, dificuldades nas relações sociais e relacionamento interpessoal, ansiedade, depressão, baixa auto-estima, desemprego, limitação quanto às atividades recreativas, dificultando, assim, o processo de adaptação do paciente.

Brown \& Kelly, apud Wellish \& Wolcott, 1994(7), descrevem oito estágios psicológicos do TMO, incluindo desde o momento de decisão do paciente para a realização do transplante até a sua adaptação fora do hospital.

A qualidade de vida de pacientes submetidos ao TMO tem emergido como uma área crítica de estudo, dadas as intensas demandas físicas e emocionais, associadas a esse procedimento, bem como o seu crescente uso, enquanto modalidade terapêutica para uma variedade de doenças malignas e hematológicas.

Desse modo, tem sido cada vez mais crescente o número de estudos sobre a satisfação e qualidade de vida desses pacientes ${ }^{(8 / 12)}$. À medida em que aumentam o interesse e a preocupação dos profissionais de 
saúde sobre qualidade de vida, aumenta também a variação existente na literatura em relação à definição e avaliação/mensuração deste conceito. Entretanto, segundo Haberman, 1993 $3^{(2)}$, há um consenso entre os pesquisadores de que qualidade de vida é um conceito multidimensional, composto por, no mínimo, quatro aspectos: bem-estar físico, psicológico, social e bem-estar espiritual.

Tschuscke, $1994^{(13)}$, observou que as estratégias de enfrentamento, utilizadas pelos pacientes, são de alto valor prognóstico, e que os dados sugerem que um coping de resignação e passividade está associado a um prognóstico pouco favorável. Por outro lado, atitudes de luta, cooperação e contato social, têm sido associadas a um processo de reabilitação mais positivo.
Nesse sentido, Andrykowski, $1995^{(1)}$, sugeriu estratégias clínicas para aumentar o ajustamento psicológico pós-TMO, enfatizando a necessidade de se promoverem expectativas reais: "a discussão de resultados pós-TMO deveria ser periodicamente reintroduzida pela equipe durante o período de recuperação, uma vez que tais expectativas constituem um foco bastante significativo para aumentar a adaptação e ajustamento psicológico pós-TMO, a longo prazo" (p.579).

Visando predizer a adaptação psicológica e o enfrentamento no processo de TMO, Wellisch \& Wolcott, 1994(7), desenvolveram medidas sistemáticas, a partir das quais o ajustamento psicossocial dos pacientes pode ser avaliado em três níveis: nível I - bom ajustamento; nível II - ajustamento regular e nível III - mau ajustamento, conforme se pode observar na Tabela I.

Tabela I - Níveis de ajustamento psicossocial de pacientes de TMO (Wellis D \& Wolcott DL, 1994) ${ }^{(7)}$ Características Psicossociais Nivel I - Bom Nível II -Regular Nível III - Mau

1 - História psiquiátrica • Nenhuma anterior

2 - Qualidade de apoio familiar/social

3 - História prévia de enfrentamento

4 - Enfrentamento da doença e tratamento

5 - Qualidade do afeto

6 - Estado mental (passado e presente)

7 - Predisposição para ansiedade antecipatória realidade.

- Nenhuma
- De bom a excelente: membros da família/meio social presentes e disponíveis.

- De boa a excelente: capacidade de adaptação aos problemas e mudanças; repertório de comportamentos de enfrentamento.

- Considerar opções de tratamento, baseando-se na

- Sentimentos de medo e tristeza apropriados; alguma ansiedade.

- Nenhum prejuízo cognitivo; • Alguma história - passada ciclo de sono preservado; nível normal de atividade (movimentos, fala).
- Alguma história de depressão, ansiedade ou trauma; traços de distúrbio de personalidade; alguma história de perda significativa.

- De bom a regular: alguma dificuldade de separação; algum conflito e problemas de dependência.

- De boa a regular: alguma flexibilidade no repertório de enfrentamento, com limitações gerais; algum padrão negativista de respostas em situações de estresse

- Negação; ambivalência na escolha do tratamento.

- Sentimentos de medo e ansiedade moderados; depressão moderada. ou atual - de prejuízo nas funções cognitivas, ciclo de sono, nível de atividade, responsividade.

- Moderada ou alta; ansiedade tem início no dia da quimioterapia/radiação.
- História de dependência química; perdas múltiplas ou significativas; grave distúrbio de ansiedade ou fobias; depressão maior.

- Disfuncional: envolvimento limitado; conflitos intensos.

- De regular a pobre: descompensação em situações de estresse; padrões negativistas; estilo rígido; história de comportamentos auto-destrutivos.

- Negação extrema; confusão no curso da doença.

- Ansiedade generalizada; depressão moderada ou grave; intensos sentimentos de medo e raiva.

- Distúrbio global das funções cognitivas; irrupção grave do ciclo de sono; nível de atividade aumentado ou reduzido.

- Grave ansiedade antecipatória; náuseas e vômitos começam antes do dia de quimioterapia/ radiação. 
De um modo geral, as pesquisas realizadas sobre a qualidade de vida pós-TMO, têm utilizado, como instrumentos de avaliação, escalas, questionários e entrevistas, apontando para padrões adaptativos gerais, deixando como questionamento as condições individuais dos sujeitos, como recurso de enfrentamento das situações envolvidas no pós-TMO.

Nesse contexto, objetivou-se avaliar as condições psicológicas e a qualidade de vida de pacientes submetidos a TMO, em dois momentos de recuperação, visando uma compreensão dos recursos disponíveis para a adaptação e o ajustamento psicossocial.

\section{MÉTODO}

\section{Sujeitos}

Procedeu-se à avaliação psicológica de dez (10) pacientes adultos (dezoito (18) a cinqüenta e três (53) anos), do sexo masculino, submetidos ao TMO, no Hospital das Clínicas da Faculdade de Medicina de Ribeirão Preto-USP. Foram estabelecidos os seguintes critérios de inclusão: Grupo 1 - Adaptação inicial - ter de três a quatro meses de seguimento, pós-alta da enfermaria (aproximadamente seis meses pós-TMO); Grupo 2 - ter, no mínimo, doze (12) meses de seguimento, pós-alta da enfermaria (aproximadamente quinze (15) meses pós-TMO), sem intercorrências clínicas, considerando, ainda, a idade mínima de dezoito (18) anos para ambos os grupos, bem como o critério de gênero, estabelecido após o início da coleta. Todos os pacientes realizaram transplante alogênico, com tempo pós-TMO variando de seis meses a quatro anos e cinco meses, sendo seis pacientes transplantados com diagnóstico inicial de Leucemia Mielóide Crônica e quatro pacientes com diagnóstico de Anemia Aplástica Severa. Observou-se um predomínio de baixo nível de escolaridade, bem como atividades profissionais de pouca qualificação, estando a maioria inativa no momento da avaliação.

\section{Instrumentos}

Para a coleta de dados, foram utilizados os instrumentos, relacionados a seguir.

- Questionário de História Vital, abordando dados de identificação (cor, idade, sexo, escolaridade, religião, estado civil, profissão, procedência).

- Questionário sobre Qualidade de Vida e Recuperação, com base nas proposições de Haberman et al; $1993^{(2)}$, abordando questões relativas ao estado geral de saúde e à adaptação social.
- Inventário de Ansiedade Traço-Estado-IDATE-Parte I/II, conforme Biaggio et al; $1977^{(14)}$. Trata-se de instrumento que avalia os estados emocionais transitórios e permanentes. Foi, originalmente, desenvolvido para investigar fenômenos de ansiedade em adultos normais, sem perturbações de ordem psiquiátrica. É composto de duas escalas distintas, elaboradas para medir duas dimensões de ansiedade: estado de ansiedade (A - estado) - como o indivíduo se sente num determinado momento - e traço de ansiedade (A - traço) - como o indivíduo se sente, geralmente, indicando, respectivamente, estado transitório (envolvendo sentimentos de tensão, nervosismo, preocupação e apreensão), e traço relativamente estável da personalidade. O IDATE foi adaptado e normatizado para o Brasil por Biaggio et al; $1977^{(14)}$. Dados normativos mais recentes e um estudo de validação foi desenvolvido por Pasquali et al; $1994^{(15)}$, tendo sido diferenciado, a nível estatístico, dois fatores, a saber, estresse e estabilidade emocional.

- Escala de Locus de Controle de Levenson, padronizada por Dela Coleta, $1987^{(16)}$. O constructo locus de controle é uma variável que busca explicar a percepção das pessoas sobre a fonte de controle dos acontecimentos, em que são envolvidas. A expectativa de controle constitui, portanto, um elemento que interfere no enfrentamento das situações e, conseqüentemente, na capacidade adaptativa.

A característica principal desta escala consiste em avaliar três dimensões de controle, a saber: a subescala Internalidade ( I ) mede o grau em que o sujeito acredita que mantém controle sobre sua vida, a subescala Externalidade-Outros poderosos $(\mathrm{P})$ mede a percepção de que o controle seria externo, dependente de outros poderosos, e a subescala Externalidade-Acaso (C) avalia a percepção de ser controlado pelo acaso, sorte ou destino.

- Escala de Ansiedade e Depressão para Hospital Geral, proposta por Botega et al; $1995^{(17)}$, desenvolvida para estimar a prevalência de transtornos do humor em pacientes adultos com afecções físicas, em ambientes não psiquiátricos.

\section{Procedimento de Coleta de Dados}

Os pacientes foram contactuados por telefone ou pessoalmente, durante o retorno médico. A participação no estudo foi voluntária, sendo firmada num termo de consentimento. Os instrumentos foram aplicados individualmente, por uma psicológa - a primeira autora citada neste estudo - em duas sessões de 
avaliação, que foram realizadas em um consultório da Fundação Hemocentro do Hospital das Clínicas da Faculdade de Medicina de Ribeirão Preto -USP.

\section{Tratamento dos Dados}

Com relação ao Questionário de História Vital e Questionário sobre Qualidade de Vida e Recuperação, as entrevistas foram gravadas e posteriormente transcritas. Quanto ao Questionário de Qualidade de Vida e Recuperação, as respostas foram categorizadas e os temas agrupados de acordo com a semelhança de conteúdos. Os demais instrumentos contam com estudos de padronização para a população brasileira e foram cotados segundo as recomendações de cada técnica.

Na análise e discussão dos dados, tomou-se como variável o tempo de recuperação pós-TMO: adaptação inicial - Grupo 1 - e adaptação a longo prazo - Grupo 2, considerando-se, ainda, os níveis de ajustamento psicossocial, propostos por Wellisch \& Wolcott, $1994^{(7)}$. esta foi melhorada. Vale ressaltar, no entanto, que a qualidade de vida, para a maioria dos sujeitos, é vista como a redução ou o desaparecimento dos sintomas trazidos pela doença de origem. Nesse sentido, a autoavaliação da qualidade de vida atual passa mais pelo crivo dos aspectos físicos, em detrimento dos aspectos psicossociais - como sentimentos de insegurança, incapacidade, perda da autonomia. Tal atitude, provavelmente, está associada à própria concepção que esses pacientes têm acerca do que vem a ser qualidade de vida, lembrando que a condição sócio-econômica da maioria é bastante precária - mesmo antes do surgimento da doença. Assim, a qualidade de vida talvez se restrinja ao fato de ter saúde.

Com relação às dificuldades relatadas pelos sujeitos, todos do Grupo 1 - adaptação inicial - manifestaram dificuldades no que concerne aos limites decorrentes da doença ou do tratamento, o que pode estar diretamente relacionado ao tempo pós-transplante, visto que o TMO é, ainda, uma experiência recente. Em contrapartida, a sexualidade e as relações sociais são

\section{RESULTADOS E DISCUSSÃO}

Os dados, obtidos através do Questionário sobre Qualidade de Vida e Recuperação, são apresentados na Tabela II, abordando especificamente a auto-avaliação da qualidade de vida atual e as principais dificuldades relatadas pelos sujeitos.

Quando instados a compararem a rotina atual com a rotina prévia de vida, nove (9), dentre os dez (10) pacientes, referem alterações. Desses nove, apenas um conseguiu retomar a rotina anterior (sujeito1), sendo este o paciente com maior tempo pós-TMO - quatro anos e cinco meses. Os demais não conseguiram retomar a rotina prévia e encontram-se afastados do serviço temporariamente, ou definitivamente desligados por não poderem assumir as funções próprias da profissão anterior.

Quanto à auto-avaliação da qualidade de vida atual, a grande maioria (oito pacientes) refere que
Tabela II - Auto-avaliação da qualidade de vida atual e principais dificuldades - Pacientes pós-TMO - adaptação inicial e a longo prazo

Auto-avaliação da qualidade de vida e principais dificuldades

Condição de vida - Inalterada Grupo 1

Grupo 2

Condição de vida - Melhorada

E

Condição de vida - Piorada

B, C, D

$1,2,3,4,5$

Limites decorrentes da doença ou do tratamento desta

A, B, C, D, E 1, 2, 4

Alterações na aparência e na capacidade física

B

1,2

Sentimentos de insegurança, incapacidade e dependência

Limites quanto ao trabalho e desempenho de atividades

Dificuldades econômicas

Dificuldades nas relações sociais

Dificuldades nas relações afetivas e familiares
A
2

Dificuldades quanto à sexualidade

C, E 5

A 3

B, D $\quad 1,3,5$

Grupo 1 - Casos $A$ - $E$

Grupo 2 - Casos 1 - 5 
preocupações mais presentes no Grupo 2 - adaptação a longo prazo - possivelmente pelo fato de estes pacientes já haverem superado os limites impostos pelo tratamento e, dessa maneira, terem espaço interno para se preocuparem com outros aspectos vitais.

A seguir, serão apresentados e discutidos os dados obtidos através das diferentes escalas.

Na Tabela III, são apresentados os resultados do Inventário de Ansiedade Traço - Estado - IDATE - dos dez sujeitos avaliados.

\begin{tabular}{|c|c|c|}
\hline \multirow{2}{*}{ Sujeitos } & \multicolumn{2}{|c|}{ Resultados } \\
\hline & $\begin{array}{c}\text { Estado } \\
\text { dado bruto }\end{array}$ & $\begin{array}{c}\text { Traço } \\
\text { dado bruto }\end{array}$ \\
\hline A & $29 \downarrow$ & $34 \mathrm{~N}$ \\
\hline B & $40 \mathrm{~N}$ & $42 \mathrm{~N}$ \\
\hline C & $25 \downarrow$ & $32 \mathrm{~N}$ \\
\hline D & $38 \mathrm{~N}$ & $44 \mathrm{~N}$ \\
\hline$E$ & $29 \downarrow$ & $27 \downarrow$ \\
\hline 1 & $54 \uparrow$ & $45 \mathrm{~N}$ \\
\hline 2 & $31 \mathrm{~N}$ & $31 \mathrm{~N}$ \\
\hline 3 & $28 \downarrow$ & $52 \uparrow$ \\
\hline 4 & $31 \mathrm{~N}$ & $37 \mathrm{~N}$ \\
\hline 5 & $36 \mathrm{~N}$ & $50 \mathrm{~N}$ \\
\hline
\end{tabular}

Valores Normativos:

A-Estado - 41,29 $(\sigma=10,53) \quad \mathbf{N}=$ dentro da média

A-Traço $-40,69(\sigma=10,65) \quad \uparrow=$ acima da média

Grupo 1 - Casos $A$ - $E$

$\downarrow=$ abaixo da média

Grupo 2 - Casos 1 - 5

Na avaliação através do IDATE, os resultados indicam que a maioria dos sujeitos não experimentam ansiedade, durante a situação de teste - com exceção do sujeito 1, cujo escore foi elevado na escala A-estado, sugerindo um alto nível de ansiedade que, provavelmente, está associado a sentimentos de tensão por se encontrar numa situação de avaliação, visto que seu escore na escala A-traço manteve-se dentro da média, não indicando, portanto, manifestação de ansiedade na vida diária.
Quanto à subescala A-traço, a maioria dos sujeitos obtiveram resultados que se encontram dentro da média da população geral, com exceção do sujeito $\mathbf{E}$ - cujo resultado se encontra abaixo da média - e do sujeito 3, que apresentou um escore elevado, indicando uma propensão à ansiedade, como resposta a situações percebidas como ameaçadoras.

Analisando-se qualitativamente as questões respondidas como positivas, de um modo geral, os sujeitos se perceberam como calmos, confiantes e satisfeitos. No entanto, dois sujeitos se mostraram mais sintomáticos - o sujeito $\mathbf{D}$ indica como sendo muito freqüentes os seguintes sentimentos pessoais: "Não tenho muita confiança em mim mesmo", "Deixo-me afetar muito pelas coisas", "Preocupo-me demais com coisas sem importância", "Evito ter que enfrentar crises ou problemas", "Fico tenso e perturbado, quando penso em meus problemas no momento"; O sujeito 5 indica, também, como sendo muito freqüientes os sentimentos acima descritos, além de responder positivamente ao item: "Sinto-me deprimido".

Quanto à percepção de controle sobre os acontecimentos, os dados estão apresentados na Tabela IV.

\begin{tabular}{cccc}
\multirow{2}{*}{$\begin{array}{l}\text { Tabela IV - Pacientes pós-TMO - adaptação inicial } \\
\text { e a longo prazo-avaliação através da Escala Mul- } \\
\text { tidimensional de Locus de Controle de Levenson }\end{array}$} \\
\hline \multirow{3}{*}{ Sujeitos } & \multicolumn{3}{c}{ Resultados } \\
\cline { 2 - 4 } & Escala C & Escala P & Escala I \\
\hline A & $12 \mathrm{~N}$ & $9 \downarrow$ & $37 \uparrow$ \\
B & $27 \uparrow$ & $18 \mathrm{~N}$ & $25 \mathrm{~N}$ \\
C & $28 \uparrow$ & $22 \mathrm{~N}$ & $32 \mathrm{~N}$ \\
$\mathrm{D}$ & $33 \uparrow$ & $26 \uparrow$ & $33 \mathrm{~N}$ \\
E & $34 \uparrow$ & $32 \uparrow$ & $34 \uparrow$ \\
1 & $19 \mathrm{~N}$ & $19 \mathrm{~N}$ & $34 \uparrow$ \\
2 & $14 \mathrm{~N}$ & $14 \mathrm{~N}$ & $34 \uparrow$ \\
4 & $22 \mathrm{~N}$ & $24 \uparrow$ & $27 \mathrm{~N}$ \\
5 & $33 \uparrow$ & $26 \uparrow$ & $32 \mathrm{~N}$ \\
\hline
\end{tabular}

Valores Normativos:

Escala C (externalidade-acaso) - 17,4 $(\sigma=5,3)$

Escala P (externalidade-outros poderosos) - 17,8 $(\sigma=5,0)$

Escala I (internalidade) - 28,7 $(\sigma=4,3)$

$\mathbf{N}=$ dentro da média

$\uparrow$ = acima da média

$\downarrow=$ abaixo da média

Grupo 1 - Casos $A$ - E

Grupo 2 - Casos 1 - 5 
Os valores obtidos nas diferentes subescalas do Locus de Controle de Levenson indicam que a externalidade esteve mais acentuada nos pacientes do Grupo 1 - adaptação inicial - predominando a tendência em explicar as ocorrências mais externamente, onde os acontecimentos da vida diária seriam atribuídos ao acaso, ao azar, ao destino, à vontade de Deus ou à influência de "pessoas poderosas"; tal atitude, possivelmente, está associada à dependência que a maioria destes pacientes assumem, ao delegarem a responsabilidade de sua evolução à equipe médica e também à crença sempre presente de que estão curados "graças a Deus". Não obstante, a externalidade - presente em ambos os grupos - não exclui a percepção dos sujeitos em se verem como controladores dos fatos que ocorrem em suas vidas, uma vez que todos os escores obtidos na subescala I encontram-se superiores ou dentro da média da população geral. Depreende-se, portanto, a situação conflitiva da maioria dos sujeitos, no que diz respeito as suas percepções sobre a fonte de controle dos acontecimentos em que são envolvidos.

Os dados relativos às manifestações de ansiedade e depressão são apresentados na Tabela V.

Tabela V - Pacientes pós-TMO - adaptação inicial e a longo prazo - avaliação através da Escala de Ansiedade e Depressão para Hospital Geral

\begin{tabular}{ccc}
\hline \multirow{2}{*}{ Sujeitos } & \multicolumn{2}{c}{ Resultados } \\
\cline { 2 - 3 } & Ansiedade & Depressão \\
\hline A & $5 \mathrm{~N}$ & $1 \downarrow$ \\
B & $6 \mathrm{~N}$ & $1 \downarrow$ \\
C & $3 \mathrm{~N}$ & $1 \downarrow$ \\
D & $3 \mathrm{~N}$ & $5 \mathrm{~N}$ \\
E & $0 \downarrow$ & $7 \mathrm{~N}$ \\
1 & $10 \mathrm{~N}$ & $6 \mathrm{~N}$ \\
2 & $3 \downarrow$ & $1 \downarrow$ \\
3 & $6 \mathrm{~N}$ & $10 \mathrm{~N}$ \\
4 & $2 \downarrow$ & $3 \mathrm{~N}$ \\
5 & $9 \mathrm{~N}$ & $3 \mathrm{~N}$ \\
\hline
\end{tabular}

Valores Normativos:

Escala de Ansiedade 7,7 $(\sigma=4,3) \quad \mathbf{N}=$ dentro da média

Escala de Depressão $7,3(\sigma=4,8) \quad \uparrow=$ acima da média

$\downarrow=$ abaixo da média

Grupo 1 - Casos $A$ - $E$

Grupo 2 - Casos 1 - 5
As pontuações alcançadas, na comparação com os dados normativos, indicam ausência de sintomas depressivos e de ansiedade para todos os sujeitos avaliados nos dois grupos.

Visando compreender melhor os níveis de ajustamento psicossocial dos pacientes submetidos ao TMO, integraram-se os dados das diferentes técnicas, no sentido de caracterizar os níveis de ajustamento, conforme o proposto por Wellisch \& Wolcott, 1994(7). Estes dados estão descritos na Tabela VI.

Segundo os autores, das características psicossociais citadas, as mais importantes na predição de problemas são as três primeiras. Relatam que as reações mais freqüentes são: ansiedade - que pode ser mais grave durante a fase de enxertamento da medula, devido ao medo consciente de morte; aflição/tristeza - freqüentemente confundidas com depressão, por apresentarem sinais e sintomas semelhantes, não se tratando, contudo, de um desajustamento e, sim, de um ajustamento funcional; regressão - nível mais baixo de integração, ajustamento e expressão, principalmente durante a fase de condicionamento, quando o impacto é mais intenso; ambivalência - atitudes de hostilidade, devidas ao trauma, dor e sofrimento induzidos pelo TMO; desregulação neuropsiquiátrica - onde alta dose de ciclofosfamida tem sido associada com euforia, delírio e sentimentos de irrealidade.

Analisando-se os dados relativos à história psiquiátrica anterior, observou-se que a maioria dos pacientes apresentaram indicadores de dificuldades, incluídos nessa categoria, tais como perdas múltiplas ou significativas e história de dependência química. Considerando-se as demais características psicossociais, observa-se que apenas os sujeitos D (nível II) e 4 (nível III) apresentaram um maior comprometimento, sugerindo que a história psiquiátrica anterior não foi, dentro dos limites deste estudo, uma variável que discriminou com clareza o nível de ajustamento desses pacientes no pós-TMO. Esse dado contraria a assertiva dos autores ${ }^{(7)}$, uma vez que os mesmos caracterizam essa variável psicossocial como altamente preditora do nível de adaptação, em pacientes submetidos ao TMO.

Convém salientar que, embora a qualidade de apoio familiar da maioria dos pacientes tenha se mostrado satisfatória, o desempenho social dos mesmos é bastante restrito, uma vez que a maioria passa grande parte do tempo assistindo à televisão ou auxiliando nos afazeres domésticos. Nenhum paciente mencionou o fato de engajar-se em atividades recreativas ou 
Tabela VI - Níveis de ajustamento psicossocial de pacientes pós-TMO - adaptação inicial e a longo prazo

\begin{tabular}{|c|c|c|c|}
\hline Características psicossociais & Nível I - Bom & Nivel II - Regular & Nivel III - Mau \\
\hline \multirow[t]{2}{*}{1 - História psiquiátrica anterior } & $\mathrm{B}, \mathrm{C}$ & $A, D, E$ & - \\
\hline & 3,5 & 1,2 & 4 \\
\hline \multirow[t]{2}{*}{2 - Qualidade de apoio familiar/social } & $\mathrm{C}, \mathrm{B}, \mathrm{E}$ & $\mathrm{D}$ & A \\
\hline & $1,3,5$ & 4 & 2 \\
\hline \multirow[t]{2}{*}{3 - História prévia de enfrentamento } & $E$ & $A, B, C, D$ & - \\
\hline & 5 & 1,3 & 2,4 \\
\hline \multirow[t]{2}{*}{4 - Enfrentamento da doença e tratamento } & $E$ & $B, D$ & $A, C$ \\
\hline & 4,5 & 1,2 & 3 \\
\hline \multirow[t]{2}{*}{5 - Qualidade do afeto } & - & $\mathrm{B}, \mathrm{C}, \mathrm{D}, \mathrm{E}$ & A \\
\hline & - & $1,2,4,5$ & 3 \\
\hline \multirow[t]{2}{*}{6 - Estado mental (passado e presente) } & $\mathrm{B}, \mathrm{C}, \mathrm{D}, \mathrm{E}$ & - & A \\
\hline & $1,3,5$ & 2 & 4 \\
\hline \multirow[t]{2}{*}{7 - Predisposição para ansiedade antecipatória } & $\mathrm{B}, \mathrm{C}$ & $\mathrm{D}, \mathrm{E}$ & A \\
\hline & 2 & $1,3,5$ & 4 \\
\hline
\end{tabular}

Grupo 1 - Casos $A$ - E

Grupo 2 - Casos 1 - 5

mesmo de ter um contato social mais amplo, com exceção do paciente 1, que desenvolve atividades de missionário. No entanto, é possível supor que os recursos psicossociais desses pacientes já eram limitados, antes da realização do transplante, tendo este intensificado tal situação, pelo fato de agravar ainda mais a condição sócio-econômica dos mesmos, além de cercear as possibilidades quanto ao desempenho de suas atividades profissionais e/ou escolares. Desse modo, o trabalho e/ou a escola deixam de ser, pelo menos provisoriamente, um recurso a mais na interação social.

No que diz respeito ao padrão prévio de enfrentamento, a maioria deu indícios de capacidade de adaptação aos problemas e mudanças advindos do meio, denotando alguma flexibilidade nas respostas de enfrentamento, classificando-se como ajustamento bom ou regular. No entanto, ao contrário do que sustentam Wellish \& Wolcott ${ }^{(7)}$, dentro dos limites desse estudo, essa variável psicossocial apresentou baixa potencialidade na predição quanto aos problemas no pós-TMO, visto que os pacientes que tinham um repertório prévio de enfrentamento, avaliado como pobre, reagi- ram à doença e ao tratamento de forma positiva, com ajustamento bom (4) ou regular (2). Em contrapartida, os pacientes que apresentaram uma limitação quanto às suas estratégias para lidar com a doença e o tratamento, apresentando mau ajustamento, não haviam demonstrado prejuízo no padrão prévio de enfrentamento.

Com relação à qualidade do afeto, é possível observar que a maioria dos sujeitos concentra-se no nível II, estando presentes, de maneira moderada, sentimentos de medo, ansiedade e depressão.

Quanto ao estado mental, a maioria preserva o desempenho global das funções cognitivas, tais como percepção, pensamento, memória, orientação, raciocínio. No entanto, três pacientes relataram alteração dessas funções - os sujeitos A e $\mathbf{4}$ referem dificuldades de raciocínio e memória, associando-as ao TMO; o sujeito 2, por sua vez, relata uma dificuldade moderada na capacidade de lembrar e recordar, já antes da realização do TMO, tendo este agravado tal função.

Quanto à predisposição para ansiedade antecipatória, nota-se uma tendência da maioria (nível II e nível III) a se preocupar com o trabalho e com a 
situação econômica atual, que os mantém dependentes de outros familiares, lembrando que todos os sujeitos aqui avaliados são homens adultos, dentre os quais sete são casados, cabendo-lhes, anteriormente, a responsabilidade de serem os provedores da família. Preocupações com recidiva da doença também estiveram presentes, porém em menor proporção (sujeitos 1, 3 e 5).

Considerando-se o conjunto das variáveis psicossociais, a maioria dos pacientes concentra-se no nível II. Os autores afirmam que, nesse nível de ajustamento, os pacientes podem se beneficiar de algum tipo de psicoterapia de apoio e/ou esclarecimento, bem como do treino de auto-relaxamento, visto que, mormente, há perdas significativas ou dificuldades de separação. Acrescentam que a estabilidade do ambiente é indispensável para a adaptação e enfrentamento dos pacientes, independentemente de seu nível de ajustamento. Tal estabilidade deve envolver continuidade de cuidados pelos mesmos profissionais de saúde, tanto quanto possível: "quanto mais a estrutura permanecer estável, melhor será para conter as ansiedades e conflitos dos pacientes" (p. 558).

\section{COMENTÁRIOS FINAIS}

Ao analisarmos quantitativamente os dados, encontramos uma dificuldade de discriminar os recursos adaptativos entre pacientes de adaptação inicial e adaptação a longo prazo, principalmente no que diz respeito às escalas, uma vez que os pacientes não se diferenciaram em relação à população geral. Inicialmente, tinha-se uma expectativa de que os instrumentos escolhidos - padronizados e validados para a população brasileira - pudessem avaliar, de maneira mais imediata, os recursos adaptativos dos pacientes submetidos ao TMO; no entanto, fez-se necessária uma análise mais qualitativa dos resultados. Ademais, o presente trabalho conta com uma casuística muito pequena, o que dificulta uma análise quantitativa dos dados, dando a este estudo um caráter preliminar. Assim, os mesmos foram trabalhados mais no sentido de identificar as peculiaridades e diferenças dos sujeitos. Desse modo, do ponto de vista do diagnóstico das condições psicossociais, seria difícil planejar uma intervenção com base exclusivamente nos dados quantitativos desses instrumentos. A inclusão de outros instrumentos e técnicas, que avaliam aspectos mais estruturais da personalidade, poderia favorecer um estudo mais aprofundado e detalhado dos recursos disponíveis na condição pós-TMO.

Outro recurso, que facilitaria uma melhor compreensão do processo adaptativo desses pacientes, seriam estudos longitudinais, que possibilitariam a avaliação do padrão adaptativo em diferentes momentos.

Analisando-se as características psicossociais e seu valor preditor, com base na literatura, observaram-se algumas diferenças. Elas, possivelmente, estão relacionadas com as condições gerais de vida e com o contexto sócio-cultural do grupo de pacientes aqui estudados, comparativamente aos do estudo de referência ${ }^{(7)}$. Desse modo, a concepção sobre qualidade de vida também estaria sujeita ao contexto sócio-cultural.

Nesse sentido, a não discriminação dos dados, obtidos pelos sujeitos nas diferentes escalas, quando comparados com a população geral, parece corroborar a condição de TMO clinicamente bem sucedida em todos os sujeitos estudados.

Esta pesquisa conta com o apoio:

- Fundação de Amparo à Pesquisa do Estado de São Paulo (FAPESP);

- Fundação Hemocentro de Ribeirão Preto/HCRP (FUNDHERP);

- Fundação de Apoio ao Ensino, Pesquisa e Assistência do HCFMRP-USP (FAEPA).

ALMEIDAAC; LOUREIRO SR \& VOLTARELLI JC. Psychosocial adjustment and quality of life of patients submitted to bone marrow transplantation (BMT). Medicina, Ribeirão Preto, 31: 296-304, apr./june 1998.

ABSTRACT: The objective of this section is to evaluate psychological aspects and quality of life of bone marrow transplantation survivors (BMT), in two recovery moments - initial adaptation and long-term adaptation - and to search available resources to adaptation and psychosocial adjustment.

UNITERMS: Bone Marrow Transplantation. Internal-External Control. Social Adjustment. Psychological Evaluation. 


\section{REFERÊNCIAS BIBLIOGRÁFICAS}

1 - ANDRYKOWSKI MA et al. 'Returning to normal' following bone marrow transplantation: outcomes, expectations and informed consent. Bone Marrow Transplant 15: 573-581, 1995.

2 - HABERMAN M et al. Quality of life of adult long-term survivors of bone marrow transplantation: a quality analysis of narrative data. Oncol Nurs Forum 20: 1545-1553, 1993.

3 - BUSH NE et al. Quality of life of 125 adults surviving 6-18 years after bone marrow transplantation. Soc Sci Med 40: 479-490, 1995.

4 - BAKER F et al. Quality of life of bone marrow transplant longterm survivors. Bone Marrow Transplant 13: 589-596, 1994.

5 - FERREL BR et al. Nurses' perceptions of the meaning of quality of life for bone marrow transplant survivors. Cancer Nurs 18: 118-129, 1995.

6 - DECKER WA. Psychosocial considerations for bone marrow transplant recipients. Crit Care Nurs 17: 67-73, 1995.

7 - WELLISH D \& WOLCOTT DL. Psychological issues in bone marrow transplantation. In: FORMAN SJ; BLUME K \& THOMAS $E D$, eds. Bone marrow transplantation, Blackwell Scientific Publication, Boston, p. 556-571, 1994.

8 - AARONSON NK et al. The European Organization for Research and Treatment of Cancer QLQ-C30: a quality-of-life instrument for use in international clinical trials in oncology. $J$ Natl Cancer Inst 85: 365-376, 1993.
9 - BAKER F et al. Role retention and quality of life of bone marrow transplant survivors. Soc Sci Med 32: 697-704, 1991.

10 - BELEC RH. Quality of life: perceptions of long term survivors of bone marrow transplantation. Oncol Nurs Forum 19: 31-37, 1992.

11 - GRANT $M$ et al. Measurement of quality of life in bone marrow transplantation survivors. Qual Life Res 1: 375-384, 1992.

12 - KRAMER JH et al. Cognitive and adaptative behavior 1 and 3 years following bone marrow transplantation. Bone Marrow Transplant 7: 5-9, 1991.

13 - TSCHUSCHKE V et al. Coping bei knochenmarktransplantation. Psychother Psychosom Med Psychol 44: 346-356, 1994.

14 - BIAGGIO AMB et al. Desenvolvimento da forma experimental em português do Inventário de Ansiedade Traço-Estado (IDATE) de Spielberger. Arq Bras Psicol Apl 29: 31-44, 1977.

15 - PASQUALI L et al. Contribuição à validação e normatização da Escala de Ansiedade-Traço do IDATE. Psicol Teor Pesqui, 10: 411-420, 1994.

16 - DELA COLETA M F. Escala multidimensional de Locus de Controle de Levenson. Arq Bras Psicol 39: 79-97, 1987.

17 - BOTEGA NJ et al. Transtornos do humor em enfermaria de clínica médica e validação da escala de ansiedade e depressão. Rev Saúde Pública 29: 355-363, 1995.

Recebido para publicação em 23/12/97

Aprovado para publicação em 06/05/98 